\title{
Space and time connections
}

The formation and recall of an episodic memory of an object rely on the integration of information about the spatial location of the object and when it was encountered. Moreover, recall of episodic memory requires interactions between the hippocampus and the medial prefrontal cortex (mPFC), although the neural connections mediating these interactions are not well characterized. Now, Warburton and colleagues show in rats that projections to the MPFC from the dorsal and intermediate hippocampal CA1 (dCA1 and iCA1) are necessary for the temporal and spatial aspects of episodic memories, respectively.

The authors used a behavioural task to assess both the spatial and temporal aspects of episodic memory retrieval in rats. In the first phase of the task, rats explored two novel objects. In the second phase of the task, an hour later, they were separately exposed to two other novel objects. Another hour later, in the test phase, the rats encountered all four objects again, together; however, two of the four objects (one from each of the two previous phases) were placed in novel locations.

To determine whether interactions between CA1 and the MPFC are necessary for episodic retrieval, the authors temporarily disrupted the function of these regions during the test phase by injecting the AMPA receptor antagonist NQBX either into the $\mathrm{mPFC}$ of one hemisphere and the contralateral CA1, or unilaterally into the mPFC and the ipsilateral CA1. Rats usually spend more time exploring objects that they have encountered less recently or those in a novel location. When hippocampal-mPFC interactions were disrupted only in one hemisphere, the rats spent most time exploring the object from the initial phase that was in a novel location and spent the least time exploring the object from the second phase that retained its original position (the rats spent the same amount of time exploring the other objects). By contrast, when hippocampal-mPFC interactions were disrupted in both hemispheres, the rats showed no preference for any of the objects. Thus, hippocampal-mPFC interactions are necessary for the retrieval of spatial and temporal information associated with an encoded object.

Previous studies have identified projections to the $\mathrm{MPFC}$ from the dCA 1 and iCA1. Here, the authors used a virus-based system to retrogradely express lac $Z$ in projections to the $\mathrm{mPFC}$ of naive rats. They then selectively inactivated $\mathrm{dCA} 1 \rightarrow \mathrm{mPFC}$ or $\mathrm{iCA} 1 \rightarrow \mathrm{mPFC}$ neurons by injecting the dCA1 or iCA1 with the prodrug Daun02, which is converted to daunorubicin in lacZ-expressing neurons. Inactivation of $\mathrm{dCA} 1 \rightarrow \mathrm{mPFC}$ neurons impaired the ability of the animals to discriminate temporal order (that is, between objects from the first and second phases of the task); conversely, rats in which $\mathrm{iCA} 1 \rightarrow \mathrm{mPFC}$ neurons were inactivated showed deficits in memory for the original spatial contexts of the objects (that is, discriminating the novel versus the original locations of the objects).

The selectivity of the disruptive effects of inactivating $\mathrm{dCA} 1 \rightarrow \mathrm{mPFC}$ and $\mathrm{iCA} 1 \rightarrow \mathrm{mPFC}$ neurons on the temporal and spatial aspects of episodic memory, respectively, was confirmed in tasks that were designed to separately assess these memory components. Notably, inactivation of either the $\mathrm{dCA} 1 \rightarrow \mathrm{mPFC}$ or the iCA $1 \rightarrow$ mPFC neurons did not impair rats' performance in the novel-object recognition test (which tests object familiarity) or in an object-location recognition test, suggesting that, rather than affecting sensitivity to novelty, the impairments are specific to the spatial and temporal components that are associated with an object memory.

These results demonstrate that direct projections from the dCA1 and $\mathrm{iCA} 1$ to the $\mathrm{MPFC}$ are crucial for the retrieval of temporal and spatial aspects of episodic memory, and imply that the mPFC is an important hub in episodic memory.

Natasha Bray

ORIGINAL ARTICLE Barker, G. R. I. et al. Separate elements of episodic memory subserved by distinct hippocampal-prefrontal connections. Nat. Neurosci. http://dx.doi.org/10.1038/nn.4472 (2016)

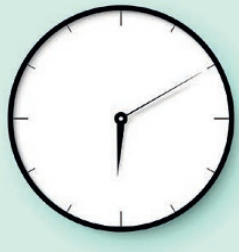

NEW YORK

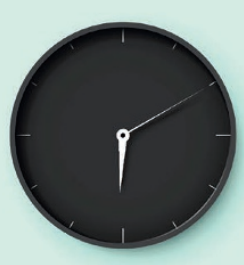

BEIJINC

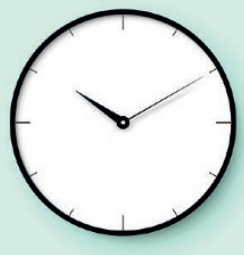

LONDON

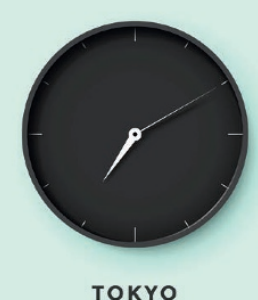

TOKYO

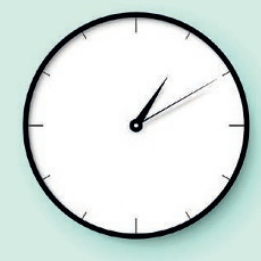

Moscow

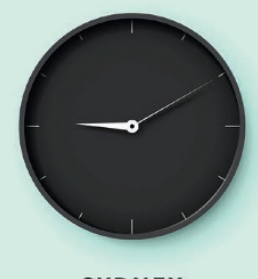

SYDNEY 